\title{
Coalition-proofness and Dominance Relations
}

\author{
Ryusuke Shinohara* \\ Graduate School of Economics, Hitotsubashi University
}

March 14, 2005

\begin{abstract}
We show that, if a game satisfies the conditions of anonymity, monotone externality, and strategic substitutability, then the set of coalition-proof Nash equilibria under strict domination contains that under weak domination.
\end{abstract}

JEL Classification Numbers: C72.

Keywords: Coalition-proof Nash equilibrium; Strict domination; Weak domination.

\section{Introduction}

This paper examines the relationship between coalition-proof Nash equilibria based on different dominance relations. The notion of a coalition-proof Nash equilibrium was introduced by Bernheim, Peleg, and Whinston (1987) and is known as a refinement of Nash equilibria based on the stability against credible coalitional deviations. However, there are two ways for a coalition to improve payoffs to its members. We consider the following two dominance relations:

\footnotetext{
*E-mail address: rusyukes@d7.dion.ne.jp
} 
(i) Strategy profile $x$ strictly dominates strategy profile $y$ if there exists a coalition $S$ such that all members of $S$ can be better off by switching $y$ to $x$, taking the strategies of the players outside $S$ as given.

(ii) Strategy profile $x$ weakly dominates strategy profile $y$ if there exists a coalition $S$ such that all members are not worse off and at least one member of the coalition is better off by deviating from $y$ to $x$, holding the strategies of the others fixed.

Under the notion of strict domination, all of the deviating players are better off, while, under that of weak domination, all members of a coalition are at least as well off, and at least one of them is better off. Thus, the set of equilibria under weak domination may be a subset of that under strict domination. This indeed applies to the strong Nash equilibrium and the core.

However, the set of coalition-proof Nash equilibria under strict domination does not contain that under weak domination. Konishi, Le Breton, and Weber (1999) provided an example in which the set of coalition-proof Nash equilibria under weak domination and that under strict domination are both non-empty and their intersection is empty. They also showed that, in the class of common agency games, any coalition-proof Nash equilibria under weak domination is that under strict domination.

In this study, we consider the class of games with $n$ players in which the strategy space of each player is a subset of the real line. ${ }^{1}$ We show that, if a game satisfies the conditions of anonymity, monotone externality, and strategic substitutability, then the set of coalition-proof Nash equilibria under weak domination is included in that under strict domination. It is interesting to point out that the same three conditions yield the equivalence of the set of coalition-proof Nash equilibria under strict domination and the weakly Pareto efficient frontier of the set of Nash equilibria (Yi (1999)). The inclusion relation between the sets of coalition-proof Nash equilibria under the two different dom-

\footnotetext{
${ }^{1}$ Note that common agency games do not belong to this class.
} 
inance relations holds for the games that have interested economists, such as standard Cournot oligopoly games and voluntary participation games in a mechanism producing public goods.

\section{The Model}

We consider a strategic game $G=\left[N,\left(X_{i}\right)_{i \in N},\left(u_{i}\right)_{i \in N}\right]$, where $N$ is a finite set of players, $X_{i}$ is the set of pure strategies of player $i$ that is a subset of real numbers, and $u_{i}: \prod_{j \in N} X_{j} \rightarrow \mathbb{R}$ is the payoff function of player $i$. In this paper, we focus solely on pure strategy equilibria. Before the coalition-proof Nash equilibria are defined, some notations will be introduced. For any coalition $S \subseteq N, x_{S} \in \prod_{j \in S} X_{j}$ designates a strategy profile of $S$. For any $x_{N} \in \prod_{j \in N} X_{j}, x_{N}$ is denoted by $x$. For any set of players $S,-S$ represents the complement of $S$.

The notions of coalition-proof Nash equilibria are defined under strict domination and weak domination. First, restricted games are introduced. For any coalition $S \subseteq N$ and any strategy profile of the complement of $S, \bar{x}_{-S}$, denote the game restricted by $\bar{x}_{-S}$ by $G \mid \bar{x}_{-S}$ in which $S$ is the set of players, $\prod_{j \in S} X_{j}$ is the set of pure strategy profiles, and $u_{i}\left(\cdot, \bar{x}_{-S}\right): \prod_{j \in S} X_{j} \rightarrow \mathbb{R}$ is player $i$ 's payoff function. Now, the definitions of coalition-proof Nash equilibria are provided under the different dominance relations.

Definition 1 A coalition-proof Nash equilibrium under weak domination is defined inductively with respect to the number of players $n$ in the game:

(i) If $n=1$, then $x_{1}^{*} \in X_{1}$ is a coalition-proof Nash equilibrium under weak domination if and only if $u_{1}\left(x_{1}^{*}\right) \geq u_{1}\left(\widetilde{x}_{1}\right)$ for any $\widetilde{x}_{1} \in X_{1}$.

(ii) Let $n>1$. Assume that the coalition-proof Nash equilibria under weak domination have been defined for games with fewer than $n$ players. 
(a) For any game $G$ with $n$ players, $x^{*} \in \prod_{j \in N} X_{j}$ is a self-enforcing strategy profile under weak domination if, for all $S \subsetneq N, x_{S}^{*} \in \prod_{j \in S} X_{j}$ is a coalition-proof Nash equilibrium under weak domination of the reduced game $G \mid x_{-S}^{*}$.

(b) Profile $x^{*}$ is a coalition-proof Nash equilibrium under weak domination of $G$ if it is a self-enforcing strategy profile under weak domination and there is no other self-enforcing strategy profile under weak domination $\widehat{x} \in \prod_{j \in N} X_{j}$ such that $u_{i}(\widehat{x}) \geq u_{i}\left(x^{*}\right)$ for all $i \in N$ and $u_{i}(\widehat{x})>u_{i}\left(x^{*}\right)$ for some $i \in N$.

Definition 2 The definition of coalition-proof Nash equilibria under strict domination is derived from Definition 1 by substituting "strict domination" for "weak domination".

Note that, in any two-player game, the set of coalition-proof Nash equilibria under weak domination coincides with the (strictly) Pareto efficient frontier of the set of Nash equilibria, and so does the set of coalition-proof Nash equilibria under strict domination with the weakly Pareto efficient frontier of that of Nash equilibria. Therefore, the set of coalition-proof Nash equilibria under weak domination is a subset of that under strict domination in two-player games. However, Konishi, Le Breton, and Weber (1999) pointed out that the inclusion relation does not necessarily hold in games with more than two players. For details, refer to Konishi, Le Breton, and Weber (1999).

\section{Results}

In this section, we establish sufficient conditions under which the set of coalitionproof Nash equilibria under weak domination is a subset of that of a coalition-proof Nash equilibrium under strict domination.

The first condition is that of anonymity. 
Anonymity. For all $i \in N$, all $x_{i} \in X_{i}$, and all $x_{-i}, \widehat{x}_{-i} \in \prod_{j \neq i} X_{j}$, if $\sum_{j \neq i} x_{j}=$ $\sum_{j \neq i} \widehat{x}_{j}$, then $u_{i}\left(x_{i}, x_{-i}\right)=u_{i}\left(x_{i}, \widehat{x}_{-i}\right)$.

The anonymity condition means that the payoff function of every player depends on his strategy and on the aggregate strategy of all other players.

The next condition is that of monotone externality. The condition states that the payoffs to every player are either non-increasing or non-decreasing with respect to the sum of strategies of the other players.

Monotone externality. For all $i \in N$, all $x_{i} \in X_{i}$, and all $x_{-i}$ and $\widehat{x}_{-i} \in \prod_{j \neq i} X_{j}$, if $\sum_{j \neq i} x_{j}>\sum_{j \neq i} \widehat{x}_{j}$, then either $u_{i}\left(x_{i}, x_{-i}\right) \geq u_{i}\left(x_{i}, \widehat{x}_{-i}\right)$ or $u_{i}\left(x_{i}, x_{-i}\right) \leq u_{i}\left(x_{i}, \widehat{x}_{-i}\right)$ holds. If the former holds, the condition means positive externalities, and it represents negative externalities if the latter is satisfied.

The third condition is that of strategic substitutability. Under this condition, the incentive of every player to reduce his strategy gets higher as the sum of the other players' strategies increases.

Strategic substitutability. For all $i \in N$, all $x_{i}, \widehat{x}_{i} \in X_{i}$, and all $x_{-i}, \widehat{x}_{-i} \in \prod_{j \neq i} X_{j}$, if $x_{i}>\widehat{x}_{i}$ and $\sum_{j \neq i} x_{j}>\sum_{j \neq i} \widehat{x}_{j}$, then $u_{i}\left(x_{i}, x_{-i}\right)-u_{i}\left(\widehat{x}_{i}, x_{-i}\right)<u_{i}\left(x_{i}, \widehat{x}_{-i}\right)-u_{i}\left(\widehat{x}_{i}, \widehat{x}_{-i}\right)$.

Before stating the main proposition, we show the following lemma.

Lemma 1 Any self-enforcing strategy profile under weak domination is that under strict domination.

Proof. Let $x$ be a self-enforcing strategy profile under weak domination of $G$. Then, by definition, $x_{C}$ is a coalition-proof Nash equilibrium under weak domination in the restricted game $G \mid x_{-C}$ for every proper subset $C$ of $N$. By the induction hypothesis, $x_{C}$ is also a coalition-proof Nash equilibrium under strict domination in $G \mid x_{-C}$. That is, for all proper subsets $C$ of $\mathrm{N}, x_{C}$ is a coalition-proof Nash equilibrium under strict 
domination of $G \mid x_{-C}$. Hence, $x$ is a self-enforcing strategy profile under strict domination of $G$.

Proposition. Suppose that a game satisfies anonymity, monotone externality, and strategic substitutability. Then, any coalition-proof Nash equilibrium under weak domination is a coalition-proof Nash equilibrium under strict domination.

Proof. If the set of coalition-proof Nash equilibria under weak domination is empty, then the statement of proposition is vacuously true. Hence, we consider the case in which there is a coalition-proof Nash equilibria under weak domination in the game. Let us assume that a game satisfies anonymity, positive externality, and strategic substitutability. ${ }^{2}$ We show by induction that the set of coalition-proof Nash equilibria under weak domination is a subset of that under strict domination. Clearly, the statement is true for all games with a single player. As stated earlier, in any two-player game, the statement is also true.

Let $n \geq 3$, and suppose that any coalition-proof Nash equilibrium under weak domination is a coalition-proof Nash equilibria under strict domination for any game with fewer than $n$ players as an induction hypothesis. Let $x^{*}$ denote a coalition-proof Nash equilibrium under weak domination of a game with $n$ players. By Lemma $1, x^{*}$ is a self-enforcing strategy profile under strict domination. We need to show that there is not other self-enforcing strategy profile under strict domination $\widetilde{x}$ where $u_{i}(\widetilde{x})>u_{i}\left(x^{*}\right)$ for every $i \in N$.

Lemma 2 There is no other self-enforcing strategy profile under strict domination $\widetilde{x}$ such that $u_{i}(\widetilde{x})>u_{i}\left(x^{*}\right)$ for all $i \in N$.

Proof of Lemma 2. Let us suppose, on the contrary, that there is a self-enforcing strategy profile under strict domination $\widetilde{x}$, at which $u_{i}(\widetilde{x})>u_{i}\left(x^{*}\right)$ for all $i \in N$. Then,

\footnotetext{
${ }^{2}$ We can similarly show the statement in the case of negative externality.
} 
$\widetilde{x}$ must satisfy the following condition.

Claim 1 It follows that $\sum_{j \neq i} x_{j}^{*}<\sum_{j \neq i} \widetilde{x}_{j}$ for all $i \in N$.

Proof of Claim 1. Suppose, on the contrary, that there is player $i \in N$ such that $\sum_{j \neq i} x_{j}^{*} \geq \sum_{j \neq i} \widetilde{x}_{j}$. By the definition of Nash equilibrium, $u_{i}\left(x^{*}\right) \geq u_{i}\left(\widetilde{x}_{i}, x_{-i}^{*}\right)$. Since $\sum_{j \neq i} x_{j}^{*} \geq \sum_{j \neq i} \widetilde{x}_{j}$ and the game satisfies anonymity and positive externality, $u_{i}\left(\widetilde{x}_{i}, x_{-i}^{*}\right) \geq u_{i}(\widetilde{x})$. Therefore, $u_{i}\left(x^{*}\right) \geq u_{i}(\widetilde{x})$, which is a contradiction. $\|$

Claim 2 The strategy profile $\widetilde{x}$ is not a Nash equilibrium of $G$.

By Claim 1, it is satisfied that $\sum_{k \in N} \sum_{j \neq k} x_{j}^{*}<\sum_{k \in N} \sum_{j \neq k} \widetilde{x}_{j}$. Hence, $\sum_{k \in N} x_{k}^{*}<$ $\sum_{k \in N} \widetilde{x}_{k}$. Therefore, $i \in N$ exists such that $\widetilde{x}_{i}>x_{i}^{*}$. By strategic substitutability, for player $i$, we have $u_{i}\left(x_{i}^{*}, \widetilde{x}_{-i}\right)-u_{i}(\widetilde{x})>u_{i}\left(x^{*}\right)-u_{i}\left(\widetilde{x}_{i}, x_{-i}^{*}\right)$. Since $x^{*}$ is a Nash equilibrium, $u_{i}\left(x^{*}\right)-u_{i}\left(\widetilde{x}_{i}, x_{-i}^{*}\right) \geq 0$. Therefore, $u_{i}\left(x_{i}^{*}, \widetilde{x}_{-i}\right)>u_{i}(\widetilde{x})$, which implies that $\widetilde{x}$ is not a Nash equilibrium of $G$. This contradicts the idea that $\widetilde{x}$ is a self-enforcing strategy profile under strict domination. Thus, there is no self-enforcing strategy profile under strict domination that dominates $x^{*}$, which implies that $x^{*}$ is a coalition-proof Nash equilibrium under strict domination in the $n$-person game.

Many interesting games in economics satisfy the conditions above. For instance, Cournot oligopoly games and the other games that have been studied as a part of industrial organization theory satisfy the conditions. For details, refer to Yi (1999). Here, we give an example in the context of the provision of pure public goods.

Example 1 Consider an economy in which there is one pure public good, one private good, $n$ players, and a mechanism that implements the Lindahl allocation rule. ${ }^{3}$ Saijo and

\footnotetext{
${ }^{3}$ Many authors have constructed the mechanisms that attain the Lindahl allocation in their equilibrium. See Tian (2000), for example.
} 
Yamato (1999) introduced a model of voluntary participation in a public good mechanism. Their model consists of two stages. In the first stage, players decide simultaneously whether or not they will participate in the public good mechanism. In the second stage, only the participants play the mechanism. Following the rule of the mechanism, they produce the public good and distribute its cost. On the other hand, non-participants can enjoy the public good produced by the participants at no cost. As a result, the participants bear the cost of the public good, but the non-participants can free-ride the public good.

In this example, we suppose that the preference relations of all players are represented by the same quasi-linear utility function $u_{i}\left(z, t_{i}\right)=\alpha \sqrt{z}+t_{i}$, where $\alpha>0, z \geq 0$ denotes the public good and $t_{i} \leq 0$ represents a payment of $i$ for producing the public good. We assume that one unit of the public good is provided from one unit of the private good. We fix an outcome of the mechanism as the Lindahl allocation only for the preferences of participants.

First, we will characterize the equilibrium outcome of the second stage. Let $p$ be the number of participants in the mechanism. The equilibrium allocation of the second stage, when $p$ players enter the mechanism, is denoted by $\left(z^{p}, t_{1}^{p}, \ldots, t_{n}^{p}\right)$. Then, the public good provision $z^{p}$ maximizes the total surplus of participants $p \alpha \sqrt{z}-z$. Hence, $z^{p}=(\alpha p / 2)^{2}$. Since every participant shares the cost of $z^{p}$ equally, every participant $i$ pays $t_{i}^{p}=-\alpha^{2} p / 4$. On the other hand, $t_{j}^{p}=0$ for every non-participant $j$. If the payoffs of participants and non-participants are denoted by $u^{1}(p)$ and $u^{0}(p)$, respectively, then we have $u^{1}(p)=\alpha^{2} p / 4$ and $u^{0}(p)=\alpha^{2} p / 2$.

Given the equilibrium outcome of the second stage, the participation decision stage can be reduced to the following simultaneous game. In the game, each player $i$ chooses either $x_{i}=1$ (participation) or $x_{i}=0$ (non-participation), simultaneously. In this stage, the set of actions of player $i$ is $X_{i}=\{0,1\}{ }^{4}$ Let $p^{x}$ be the number of participants at

\footnotetext{
${ }^{4}$ Only the pure strategies are considered.
} 
an action profile $x=\left(x_{1}, \ldots, x_{n}\right)$. Then, $p^{x}$ participants obtain the utility $u^{1}\left(p^{x}\right)$, and $n-p^{x}$ non-participants have the payoff $u^{0}\left(p^{x}\right)$ at the action profile $x$. The payoff matrix of the participation stage game in the case of $n=3$ appears in Table 1 , in which agent 1 chooses rows, agent 2 chooses columns, and agent 3 chooses matrices. The first entry in each box is agent 1's payoff, the second is agent 2's, and the third is agent 3's. We can easily check that the participation decision game satisfies the conditions of anonymity, positive externality, and strategic substitutability. In this example, two agents choose participation in every coalition-proof Nash equilibrium under weak domination, while one agent or two agents participate in the mechanism in coalition-proof Nash equilibria under strict domination.

\section{Acknowledgements}

I would like to thank Koichi Tadenuma for his helpful comments, suggestions, and continuous support. I am also grateful to Hideo Konishi for useful discussions. I wish to thank an anonymous referee. This research was supported by the 21st Century Center of Excellence Project on the Normative Evaluation and Social Choice of Cotemporary Economic Systems. Any remaining errors are my own.

\section{References}

Bernheim, D., B. Peleg, and M. Whinston, 1987, Coalition-proof Nash equilibria I: Concepts, Journal of Economic Theory 42, 1-12.

Konishi, H., M. Le Breton, and S. Weber, 1999, On coalition-proof Nash equilibria in common agency games, Journal of Economic Theory 85, 122-139.

Saijo, T., and T. Yamato, 1999, A voluntary participation game with a non-excludable public good, Journal of Economic Theory 84, 227-242. 
Tian, G., 2000, Double implementation of Lindahl allocations by a pure mechanism, Social Choice and Welfare 17, 125-141.

Yi, S., 1999, On the coalition-proofness of the Pareto frontier of the set of Nash equilibria, Games and Economic Behavior 26, 353-364. 


\begin{tabular}{|c|c|c|}
\hline & 1 & 0 \\
\hline 1 & $\frac{3 \alpha^{2}}{4}, \frac{3 \alpha^{2}}{4}, \frac{3 \alpha^{2}}{4}$ & $\frac{\alpha^{2}}{2}, \alpha^{2}, \frac{\alpha^{2}}{2}$ \\
\hline 0 & $\alpha^{2}, \frac{\alpha^{2}}{2}, \frac{\alpha^{2}}{2}$ & $\frac{\alpha^{2}}{2}, \frac{\alpha^{2}}{2}, \frac{\alpha^{2}}{4}$ \\
\hline
\end{tabular}

1

\begin{tabular}{|c|c|c|}
\hline & 1 & 0 \\
\hline 1 & $\frac{\alpha^{2}}{2}, \frac{\alpha^{2}}{2}, \alpha^{2}$ & $\frac{\alpha^{2}}{4}, \frac{\alpha^{2}}{2}, \frac{\alpha^{2}}{2}$ \\
\hline 0 & $\frac{\alpha^{2}}{2}, \frac{\alpha^{2}}{4}, \frac{\alpha^{2}}{2}$ & $0,0,0$ \\
\hline
\end{tabular}

0

Table 1: Payoff matrix of the participation decision game with $n=3$. (Example 1) 